SAND90-7091

DE9 1004984

TTC 1016

\title{
BUDGET ALLOCATION AND THE ANALYTIC HIERARCHY PROCESS`
}

\author{
Bernie L. Hulme \\ Hulme Mathematics \\ 3701 General Patch, N.E. \\ Albuquerque, New Mexico 87111
}

October 1990

\begin{abstract}
This report demonstrates that the priorities calculated by the Analytic Hierarchy Process can be used as measures of benefit for budget allocation. A procedure is described that optimally allocates a budget among competing DOE waste minimization projects. The projects are compared using an analytic hierarchy already developed by Sandia National Laboratories.
\end{abstract}

This report was prepared under contract $66-7454$ for Sandia National Laboratories, Albuquerque, New Mexico. 


\section{Introduction}

Sandia National Laboratories has just finished a Test Case [2] for the DOE Waste Minimization Program. The purpose was to develop criteria and methodology for evaluation and comparison of proposed process changes which could reduce waste production at DOE sites. In [2] Kjeldgaard, Saloio and Varnado proposed the use of the Analytic Hierarchy Process [4] to develop criteria against which alternative projects can be compared as to relative benefits.

The object of the present report is to show how the benefits derived from the Analytic Hierarchy Process (AHP) may be used in a benefit-cost analysis to do budget allocation. The main idea here is that the "priorities" derived from AHP are perfectly good benefit measures. They may be used along with traditional cost measures to do benefitcost analysis. In particular, optimum sets of alternative projects can be chosen to provide the maximum benefit possible within a given budget.

\section{The Analytic Hierarchy Process}

The Analytical Hierarchy Process, due to Thomas Saaty, is fully explained in $[4,6]$, and its recommended application to DOE Waste Minimization is summarized in [2]. Here a brief overview of AHP is given to fix terminology and to provide some background for the discussion.

\subsection{General Description}

AHP involves constructing a tree of criteria and sub-criteria (a hierarchy) for measuring how well any project achieves a given goal. The root node is the goal. Below the root node, and connected to it by arcs, are the major criteria involved in achieving the goal. Each criterion can be broken into subcriteria, represented by lower level nodes attached to their parent criterion node by arcs. Sub-criteria nodes may give rise to sub-sub-criteria nodes, etc. Finally, all of the alternatives (projects) are replicated as descendant nodes under every lowest-level criterion node. The projects are the leaves of the tree.

Having developed the relevant criteria, the use of AHP next undertakes to derive priorities for each of the alternatives being compared. Priorities are positive real numbers which reflect the relative values of the alternatives with respect to achieving the goal. Larger priority is better, and the ratio of two priorities indicates how much better. Priority calculation is based on two kinds of pairwise comparison. First, the user compares each pair of sub-criteria under a given parent criterion, which results in local priorities indicating the relative importance of each sub-criterion with respect to its parent. This is done for every parent node from the goal on down. Finally, the user does a pairwise comparison of all alternatives as to how well they rate with respect to each of the lowest-level criteria in the tree. Synthesis (weighted averaging) then produces the global priorities, which are the relative values of the alternatives with respect to achieving the goal. These are the numbers which may be taken as measures of benefit.

AHP allows the user to incorporate both objective data and subjective judgments in the pairwise comparisons. There is no need to convert judgments into monetary measures of merit. The global priorities combine all of the user's data and feelings about the relative importance of a variety of criteria and alternatives into summary measures of relative benefit. The entire process, including the calculations, has been implemented in a personal computer software package called Expert Choice [5]. 


\subsection{Local Priorities}

Local priorities are computed for the sub-criteria under each parent criterion. They indicate the relative importance of the sub-criteria with respect to the parent criterion. Local priorities are positive real numbers which sum to one. Their ratios indicate how much more important one subcriterion is than another as far as the parent criterion is concerned.

Suppose for a moment that we already know the local priorities or weights $w_{i}, 1 \leq i \leq n$, for $n$ sub-criteria with respect to a parent criterion. We could form a matrix $\mathrm{A}$ of weight ratios,

$$
A_{i j}=w_{i} / w_{j}, \quad 1 \leq i, j \leq n,
$$

where each $\mathrm{A}_{\mathrm{ij}}$ indicates how much more important sub-criterion $i$ is than $j$. If we form the column vector of weights

$$
\underline{w}-\left[w_{1}, w_{2}, \ldots, w_{n}\right]^{T} \text {, }
$$

then multiplying the weight-ratio matrix $A$ by the weight vector $\underline{w}$ yields

$$
A \underline{w}=n \underline{w},
$$

indicating that $\underline{w}$ is an eigenvector of $A$ having eigenvalue $n$. As Saaty proves from the theory of positive matrices, $n$ is the largest eigenvalue of $A$ and the $n-1$ other eigenvalues are zero.

Now suppose that we know the matrix A, but not the vector $\underline{w}$. Can we recover the weights $w_{i}$ knowing only their ratios? The answer is yes, to within a constant multiplier. By computing the dominant eigenvector of $\mathrm{A}$ and scaling it so that $w_{i}+\ldots+w_{n}=1$, we have the relative weights $w_{i}$. It is characteristic of eigenvectors that they are unique only up to a multiplier, and so they may be scaled (multiplied by a positive constant) in any convenient way. This is perfect for AHP purposes because relative weights are all we need, i.e., weights whose ratios are unique.

That is the theory behind local priority calculation. In practice, the AHP user constructs an approximate weight-ratio matrix $A$ by making numerical responses to pairwise comparisons of sub-criteria $i$ and $j$, $1 \leq i \leq n-1, i+1 \leq j \leq n$. The diagonal elements of $\mathrm{A}$ are always 1 , and the elements below the diagonal are reciprocals of the corresponding elements above the diagonal, $A_{i j}=$ $1 / A_{j i}$. The dominant eigenvalue $\lambda_{\max }$ of $A$ will equal $n$ if and only if $A$ is perfectly consistent, a condition that need not hold for AHP to be useful. The degree to which $\lambda_{\max }$ differs from $\mathrm{n}$ is a measure of the user's consistency, and Expert Choice computes a consistency index for each matrix A. If it is too high, the user can reconstruct $A$. The dominant eigenvector of $A$, scaled to have a unit sum, is an estimate of the local priorities.

Saaty points out $[4$, p.223] that local priorities belong to a ratio scale. This means they are comparable to one another, and they may be multiplied by any positive constant without losing their relative meaning; i.e., their ratios are invariant.

\subsection{Global Priorities}

The pairwise comparison of alternatives with respect to each lowestlevel criterion also leads to local priorities for the alternatives. These may be viewed as scores for the alternatives on each criterion. The global priorities, then, are overall scores for the alternatives.

Synthesizing the global priorities from the local priorities is simply a matter of forming a weighted average of an alternative's scores over all the lowest-level criteria. In the process, each lowest-level criterion's local priority is multiplied by its parent's local 
priority, which in turn is multiplied by its parent's local priority, etc., so that the lowest-level criteria are appropriately weighted with respect to the goal. Consequently, the global priority of an alternative accurately reflects the user's composite judgment about an alternative's relative importance with respect to the goal.

As for local prioriti s, global priorities belong to a ratio scale. They may be multiplied by an arbitrary positive constant, and their ratios, which indicate relative importance, will be unchanged. Global priorities are comparable to one another and provide good measures of relative benefit for competing projects.

\section{Budget Allocation with AHP Results}

\subsection{Overview}

The following procedure is recommended for maximizing the benefits achievable within a given budget when choosing among alternative waste minimization projects. Each step is further discussed below.

1. Define competing alternatives. Include a theoretical neutral alternative which corresponds to doing nothing.

2. Use AHP as recommended in [2] to derive global priorities for all the alternatives. These will serve as benefits, $b_{i}$.

3. Discard alternatives $i$ that are no better than doing nothing, i.e., $b_{i} \leq b_{\text {neut }}$.

4. Compute the costs, $c_{i}$, of the remaining alternatives using doliars as usual.

5. For a given budget $B$, solve the zere-one knapsack problem corresponding to $b_{i}$, $c_{i}, B$.

\subsection{Defining Alternatives}

It is crucial that the competing alternatives be independent. This means that they must have no benefits in common and no cost savings that accrue when doing two or more alternatives. Only when this condition holds will the benefits and costs be additive, i.e., the benefit (cost) of any two or more alternatives will be the sum of the individual benefits (costs).

For comparison purposes, include a neutral alternative which corresponds to doing nothing. Its priority will help weed out alternatives that ought not to be considered.

\subsection{Benefit Measures}

The authors of [2] have recommended using a special variation of AHP, available as an option in Expert Choice. When there is a large number of competing alternatives, the Rating Module saves the user time. Instead of listing all of the alternatives under each lowest-level criterion and repeatedly making a large number of pairwise comparisons, the user devises a few "ratings" (good, bad, poor, etc.) appropriate to each lowest-level criterion. Only the few ratings are compared pairwise, and Expert Choice assigns local priorities to the ratings. Then the user rates every alternative with respect to each criterion, and an alternative receives the local priority assigned to that rating. Synthesis proceeds as usual using these local priorities.

When using the Rating Module within this budget allocation procedure, every set of ratings must include a neutral rating meaning "no effect." The neutral alternative must receive the neutral rating under every criterion.

The key to this whole approach to budget allocation is that the global priorities used as benefits are additive. 
As Saaty points out in [4, p.244], "We may add elements from the same ratio scale to obtain a third element which belongs to the same ratio scale." Since actual benefits, $a_{i}$, are proportional to our priority measures, $b_{i}$, we have

$$
\begin{aligned}
a_{i} & =\alpha b_{i}, a_{j}=\alpha b_{j} \text { and } a_{i}+a_{j} \\
& =\alpha\left(b_{i}+b_{j}\right) .
\end{aligned}
$$

where the multiplier of the sum is still $\alpha$. Thus, we may ignore the multiplier a (which we do not know anyvay), and simply add priorities as if they were actual benefits.

In my opinion, this is the point at which AHP is clearly preferable to Multiattribute Utility Theory (MAU) [1]. In MAU, experts must provide both scaling functions (to adjust for incomparable scoring scales) and utility functions in order to arrive at additive utility values from the scores on disparate attributes. See [6, pp. 122-124] for Saaty's discussion of other issues, such as the need in MAU to assign absolute values to intangible criteria.

\subsection{Discarding Alternatives}

AHP provides a benefit for the neutral alternative as well as for all the others. Alternatives with no better benefit than $b_{\text {neut }}$ should not be considered for budget allocation because they cost more and produce less than doing nothing.

\subsection{Costs}

Any traditional cost computation method will suffice. The main condition is that the alternatives are independent so that their costs are additive.

\subsection{Maximizing Benefits}

Given a budget $B$, one maximizes total benefits without exceeding the budget by solving the zero-one knapsack problem:

$$
\begin{aligned}
\text { maximize } & \sum_{i=1}^{N} b_{i} x_{i} \\
\text { subject to } & \sum_{i=1}^{N} c_{i} x_{i} \leq B, \\
x_{i}=1, & \begin{array}{l}
\text { if alternative } \\
\text { i is chosen, } \\
0, \text { otherwise. }
\end{array}
\end{aligned}
$$

A good method for solving this problem when $b_{i}, c_{i}, B$ and $N$ are positive integers is given by Papadimitriou and Steiglitz [3]. Saaty [6] has considered even more general knapsack problems.

\section{An Example}

\subsection{The Zero-One Knapsack Approach}

Table 1 shows the benefits associated with ten hypothetical waste minimization projects $A-J$, which are independent of one another, and the neutral (do nothing) alternative. The benefits are global priorities derived from the analytic hierarchy constructed in [2] for assessing waste minimization proposals. This hierarchy rates the alternatives according to 50 lowest-level criteria.

Table 1

$\begin{array}{cc}\text { Alternative } & \text { Benefit } \\ \text { A } & 0.196 \\ \text { B } & 0.148 \\ \text { C } & 0.174 \\ \text { D } & 0.132 \\ \text { E } & 0.229 \\ \text { F } & 0.272 \\ \text { G } & 0.164 \\ \text { H } & 0.129 \\ \text { I } & 0.121 \\ \text { J } & 0.306 \\ \text { neutral } & 0.143\end{array}$


After discarding projects that are not better than doing nothing, we are left with the seven projects of Table 2, which shows benefits (priorities) and costs (in millions of $\$$ ).

Table 2

\begin{tabular}{|c|c|c|}
\hline Alternative & Benefit & Cost \\
\hline \begin{tabular}{lc}
1 & $(\mathrm{~A})$ \\
2 & $\mathrm{~B}$ \\
3 & $\mathrm{C}$ \\
4 & $(\mathrm{E})$ \\
5 & $(\mathrm{~F})$ \\
6 & $(\mathrm{G})$ \\
7 & $(\mathrm{~J})$ \\
& \multicolumn{1}{c}{ Totals }
\end{tabular} & $\begin{array}{l}0.196 \\
0.148 \\
0.174 \\
0.229 \\
0.272 \\
0.164 \\
0.306 \\
1.489\end{array}$ & $\begin{array}{l}12 \\
10 \\
12 \\
16 \\
22 \\
14 \\
\frac{28}{114}\end{array}$ \\
\hline
\end{tabular}

Table 3 displays, for a dozen different budgets, the optimum set of alternatives to fund as well as the corresponding, total benefit and total cost. The benefit is the maximum that is possible for that budget, found by solving the zero-one knapsack problem.

\subsection{A Common Misconception}

Everyone who thinks very long about the optimization problem (1)-(3), eventually considers ranking the alternatives by decreasing benefit/cost ratio. It is not true that selecting projects from the top of the benefit/cost ratio list until the budget is consumed will always maximize benefits within the budget constraint. The alternatives in Table 2 happen to be listed and numbered in order of decreasing benefit/cost ratio, yet in Table 3 the optimum set does not always begin with item 1 , even when 1 is within the budget. Nor is the second element always 2 , even if 2 is within the remaining budget, and so on. In fact at $B=20$, the benefit is maximized by a single project which has only the fourth-best benefit/cost ratio.

Table 3

\begin{tabular}{rccc}
\hline Budget & Qptimum Set f Alternatives & Max. Benefit & Cost \\
\cline { 3 - 4 } 10 & 2 & 0.148 & 10 \\
20 & 4 & 0.229 & 16 \\
30 & 1,4 & 0.425 & 28 \\
40 & $1,3,4$ & 0.599 & 40 \\
50 & $1,2,3,4$ & 0.747 & 50 \\
60 & $1,2,4,5$ & 0.845 & 60 \\
70 & $1,2,3,5,6$ & 0.954 & 70 \\
80 & $1,2,3,4,7$ & 1.053 & 78 \\
90 & $1,2,3,4,5,6$ & 1.183 & 86 \\
100 & $1,2,3,4,5,7$ & 1.325 & 100 \\
110 & $1,3,4,5,6,7$ & 1.341 & 104 \\
120 & $1,2,3,4,5,6,7$ & 1.489 & 114 \\
\hline
\end{tabular}




\section{REFERENCES}

[1] Keeney, R. L., and Raiffa, H., Decisions with Multiple Objectives: Preferences and Value Tradeoffs, John Wiley \& Sons, New York, 1976.

[2] Kjeldgaard, E. A., Saloio, J. H., and Varnado, G. B., Development and Test Case Application of a Waste Minimization Project Evaluation Method, SAND901179 , S a ndia $\mathrm{N}$ a tional Laboratories, Albuquerque, NM, August, 1990.
[3] Papadimitriou, C. H., and Steiglitz, K., Combinatorial Optimization: Algorithms and Complexity, Prentice-Hall, Englewood Cliffs, N.J., 1982.

[4] Saaty, T. L., The Analytic Hierarchy Process, McGraw-Hill, New York, 1980.

[5] Saaty, T. L., Forman, E. H., et al., Expert Choice manual, Expert Choice, Inc., Pittsburgh, 1989.

[6] Saaty, T. L., and Kearns, K. P., Analytical Planning, Pergamon Press, Oxford, 1985. 

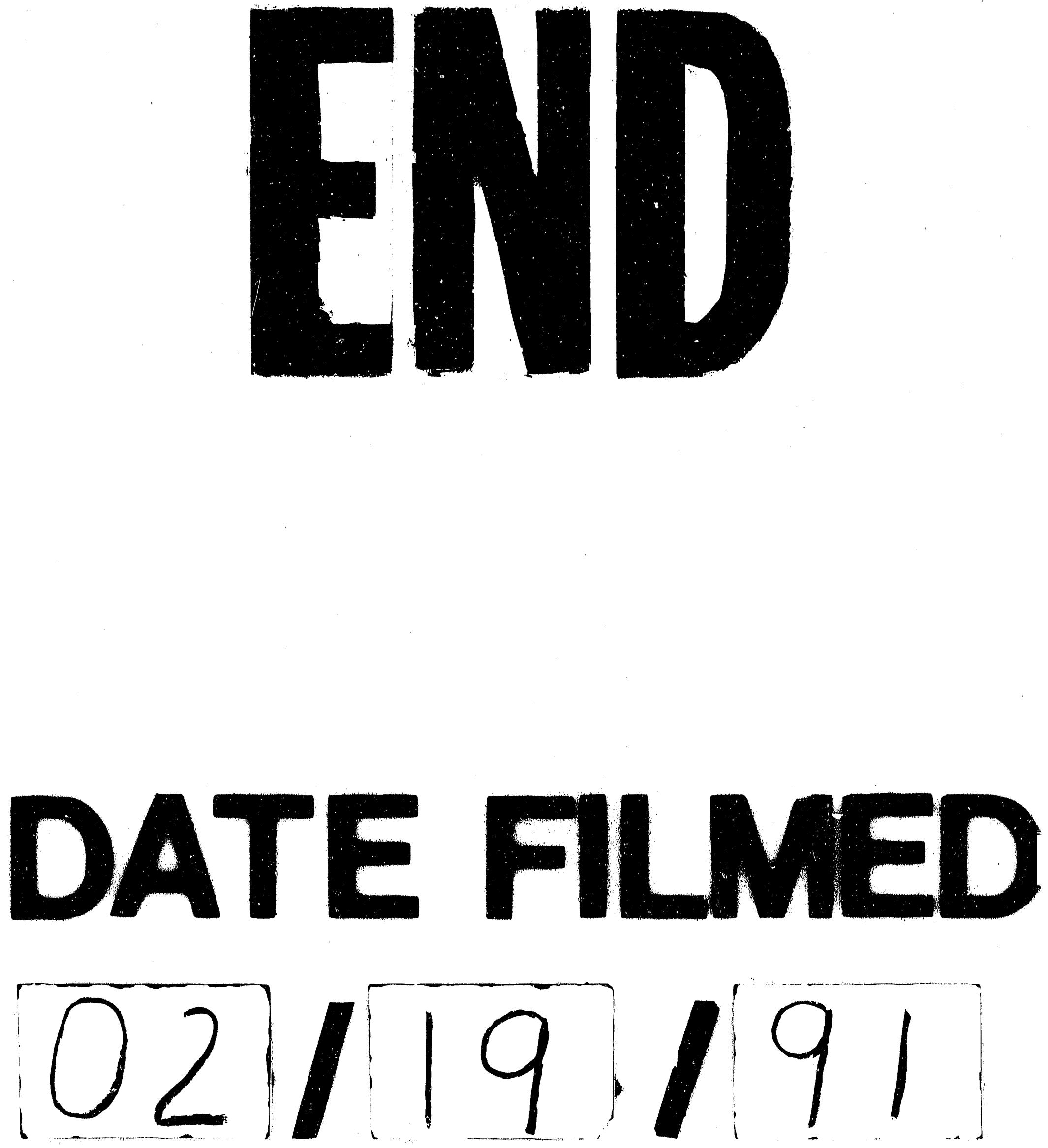
